\title{
Triglyceride-mediated influence of serum angiopoietin-like protein 8 on subclinical atherosclerosis in type 2 diabetic patients: results from the GDMD study in China
}

\author{
Tianpeng Zheng ${ }^{1 * \dagger}$, Bo Ge ${ }^{2 \dagger}$, Hongbo Liu ${ }^{3}$, Bo Chen ${ }^{4}$, Linyuan Qin ${ }^{5}$, Liuping Xiao ${ }^{1}$ and Jianfei Song ${ }^{6}$
}

\begin{abstract}
Background: Hypertriglyceridemia, insulin resistance and hyperglycemia are risk factors for atherosclerosis in type 2 diabetes. Angiopoietin-like protein 8 (ANGPTL8) is a newly identified liver-derived hormone related to these risk factors. Hence, we aimed to explore the correlations between serum levels of ANGPTL8 and subclinical atherosclerosis in type 2 diabetes.

Methods: We measured serum ANGPTL8, blood lipids, blood glucose, common carotid artery Intima-Media Thickness (c-IMT) and calculated homeostasis model assessment of insulin resistance in (1) control subjects $(n=100),(2)$ type 2 diabetic patients without subclinical atherosclerosis $(n=100)$, and (3) type 2 diabetic patients with subclinical atherosclerosis $(n=100)$.

Results: Serum levels of ANGPTL8 and triglyceride (TG) were significantly increased in type 2 diabetic patients with subclinical atherosclerosis as compared with type 2 diabetic patients without subclinical atherosclerosis and control subjects $(P<0.001)$. ANGPTL8 was positively associated with age, TG, diabetes duration, and c-IMT in type 2 diabetes. Logistic regression analysis revealed that ANGPTL8 had higher odds of having subclinical atherosclerosis [odds ratio (OR) 2.90, 95\% confidence interval (CI) 1.48-5.70, $\mathrm{P}=0.002$ ] in type 2 diabetes. Mediation analysis indicated that TG acted as a partial mediator in the relationship between ANGPTL8 and C-IMT.
\end{abstract}

Conclusions: TG partially mediates the positive relationship between ANGPTL8 and C-IMT. Our data provide the first evidence for a strong link between ANGPTL8 and subclinical atherosclerosis, suggesting ANGPTL8 to be a new biomarker for subclinical atherosclerosis in type 2 diabetes.

Keywords: ANGPTL8, Atherosclerosis, Type 2 diabetes, Triglyceride, Insulin resistance, Hyperglycemia

\section{Background}

Type 2 diabetes is a chronic multisystem disease associated with a cluster of vascular complications [1]. Atherosclerotic complications are the leading causes of morbidity and mortality among type 2 diabetic patients

\footnotetext{
*Correspondence: w19831120@126.com

TTianpeng Zheng and Bo Ge contributed equally to this work

${ }^{1}$ Department of Endocrinology and Metabolism, The Second Affiliated Hospital of Guilin Medical University, 212 Renmin Road, Guilin 541199,

Guangxi, People's Republic of China

Full list of author information is available at the end of the article
}

[2]. The detailed pathogenesis of atherosclerosis in type 2 diabetes is still not entirely clear, therefore, the identification of novel biomarkers and underlying mechanisms related to subclinical atherosclerosis in type 2 diabetes is of critical importance.

Angiopoietin-like protein 8 (ANGPTL8) (alternatively named refeeding-induced fat and liver [RIFL], lipasin, TD26) is a secreted protein of 198 amino acids primarily expressed in liver and adipose tissues. A metaanalysis study by Yue et al. revealed that type 2 diabetic patients had higher circulating levels of ANGPTL8 compared with those without type 2 diabetes, suggesting a 
relationship between increased ANGPTL8 and glucose metabolism [3]. In addition to its relationship with glucose metabolism, ANGPTL8 has also been implicated in both lipid metabolism and insulin resistance, all of which are thought to be involved in the pathogenesis of atherosclerosis [4-6]. Therefore, we were motivated to hypothesize that increased circulating levels of ANGPTL8 might be positively associated with common carotid artery Intima-Media Thickness (c-IMT) and serve as a novel biomarker for atherosclerosis in type 2 diabetes. To date, no study has ever evaluated the association between circulating levels of ANGPTL8 and c-IMT or considered the possibility of identifying ANGPTL8 as a risk biomarker for subclinical atherosclerosis in type 2 diabetes.

Consequently, the objective of the present study was to answer the three following questions: (1) Whether circulating levels of ANGPTL8 are increased in type 2 diabetic patients with subclinical atherosclerosis. (2) Is the increase in circulating levels of ANGPTL8 positively associated with subclinical atherosclerosis in type 2 diabetes? (3) If the second answer is yes, what factors mediate this positive relationship between ANGPTL8 and subclinical atherosclerosis in type 2 diabetes?

\section{Methods}

\section{Study population}

This was a cross-sectional analysis of baseline data from Guangxi Diabetes and Metabolic Disorders (GDMD) study aimed to investigate the etiology and comorbidities of type 2 diabetes and metabolic syndrome [7]. Participants were enrolled between February 2016 and May 2016 at the Medical Examination Center of the Affiliated Hospital of Guilin Medical University. A total of 300 Chinese subjects were enrolled, of which 100 control subjects with normal glucose tolerance (NGT), 100 type 2 diabetic patients with subclinical atherosclerosis and 100 type 2 diabetic patients without subclinical atherosclerosis. Exclusion criteria were as follows: (1) any evidence of acute diabetic complications, acute inflammatory diseases, malignancy, heart, liver, kidney, and respiratory failure. (2) Use of possible or known drugs affecting triglyceride (TG) for more than 3 months or at any time within 12 months before the recruitment. (3) Insulin use. (4) Subjects with incomplete data. The study was approved by the Ethics Committee of Affiliated Hospital of Guilin Medical University, and written informed consent has been obtained from each participant after full explanation of the purpose and nature of our study. This study was registered on the Chinese Clinical Trial Registry (ChiCTR-EPC-14005273).

\section{Measurements}

A standard questionnaire was administered by trained staff to the patients to record demographic and clinical data. Anthropometric parameters were measured and calculated as previously described [8]. Participants were instructed to maintain their usual diet and physical activity for at least 3 days before the examination. After an overnight fast of $10 \mathrm{~h}$ or longer, venous blood samples were collected to measure fasting plasma glucose (FPG), fasting insulin, HbA1c, blood lipids and ANGPTL8, blood samples were also drawn at $120 \mathrm{~min}$ after a $75 \mathrm{~g}$ glucose load to measure glucose in controls subjects with normal glucose tolerance. Plasma glucose levels, insulin, TC, TG, HDL-C and LDL-C were measured as previously described [1]. Serum levels of ANGPTL8 were quantified using a commercially available ELISA kit (Wuhan Eiaab Science, Wuhan, China) according to the manufacturer's instructions. The determination of c-IMT was performed as previously described [9]. The subclinical atherosclerosis was defined as c-IMT $>0.9 \mathrm{~mm}$ and/or presence of AS plaques in the carotid artery, the presence of carotid plaques was defined as focal echo structures encroaching into the arterial lumen of at least $0.5 \mathrm{~mm}$ or $50 \%$ of the surrounding c-IMT value, or when c-IMT was $>1.5 \mathrm{~mm}$ as measured from the media-adventitia interface to the intima-lumen interface [1]. The homeostasis model assessment of insulin resistance (HOMA-IR) was calculated as previously reported (HOMA-IR = Fasting glucose * Fasting insulin/22.5) [1].

\section{Statistical analysis}

Statistical analyses were conducted using SPSS 16.0 software. The distribution of continuous variables were checked for normality before analysis, TG, fasting insulin and HOMA-IR were normalized by a logarithm transformation. Continuous variables were described as mean $\pm \mathrm{SD}$, categorical variables were described as frequencies and proportions. Clinical and biochemical parameters were compared by ANOVA, Chi square, or $\mathrm{t}$ test. Partial correlation analysis and multivariate regression analysis controlling for age, BMI and gender were estimated to examine the relationship between variables. A binary logistic regression analysis was used to estimate the association of age, TG, duration of diabetes and ANGPTL8 with the odds of subclinical atherosclerosis in type 2 diabetes.

To determine whether the relationship between ANGPTL8 and c-IMT was mediated by hypertriglyceridemia, insulin resistance and hyperglycemia, mediation analysis were conducted based on the procedures outlined by Baron and Kenny [10]. A three-step linear regression model was constructed as follows: (1) $\mathrm{Y}=\mathrm{cX}+\mathrm{e} 1$ (2) $\mathrm{M}=\mathrm{aX}+\mathrm{e} 2$ (3) $\mathrm{Y}=\mathrm{c}^{\prime} \mathrm{X}+\mathrm{bM}+\mathrm{e}$, where $\mathrm{X}$ is the 
independent variable (ANGPTL8), $\mathrm{Y}$ is the dependent variable (c-IMT), $\mathrm{M}$ is the mediator, $\mathrm{a}$ is the regression coefficient for the association between ANGPTL8 and mediator, $b$ is the regression coefficient for the association between mediator and c-IMT, $\mathrm{c}$ is the regression coefficient for the association between ANGPTL8 and c-IMT, and c' is the effect of ANGPTL8 on c-IMT while controlling for the indirect effect. A Sobel Test was further performed to test mediation: First, the indirect effect was calculated by the effect of the independent variable on the mediator obtained from the first regression model multiplied by the effect of the mediator on the dependent variable from the third regression model. Second, the indirect effect was further divided by its standard error and a $\mathrm{z}$ test was performed [11]. An indirect ratio was used to present the strength of mediation: $\left(\left[\mathrm{a}^{*} \mathrm{~b}\right] / \mathrm{c}\right)$.

In this analysis, four conditions used to establish mediation were as follows: (1) the independent variable should be significantly associated with the dependent variable; (2) the independent variable should be significantly associated with the mediator; (3) the mediator should be significantly associated with the dependent variable; (4) the relationship between the dependent and independent variable should be attenuated when the mediator is included in the regression model.

\section{Results}

\section{Clinical and laboratory characteristics}

Table 1 summarized the clinical characteristics of the three groups including control subjects with normal glucose tolerance, type 2 diabetic patients without subclinical atherosclerosis, and type 2 diabetic patients with subclinical atherosclerosis. Our data indicated that statin use, TG, duration of diabetes, c-IMT and ANGPTL8 in type 2 diabetic patients with subclinical atherosclerosis had a significant increase than in control subjects with NGT and type 2 diabetic patients without subclinical atherosclerosis, whereas leisure-time physical activity had a significant decrease in type 2 diabetic patients with subclinical atherosclerosis than in control subjects. There were no significant differences in age, sex, BMI, current

Table 1 Clinical characteristics in control subjects with normal glucose tolerance (NGT), type 2 diabetes (T2DM) patients without atherosclerosis, and T2DM patients with atherosclerosis

\begin{tabular}{|c|c|c|c|c|}
\hline Characteristics & $\begin{array}{l}\text { Control subjects with NGT } \\
(n=100)\end{array}$ & $\begin{array}{l}\text { T2DM without atherosclerosis } \\
(n=100)\end{array}$ & $\begin{array}{l}\text { T2DM with atherosclerosis } \\
(n=100)\end{array}$ & $P$ value \\
\hline Age (years) & $56.4 \pm 7.6$ & $56.2 \pm 8.4$ & $57.2 \pm 7.7$ & 0.628 \\
\hline Percent men (\%) & 54 & 56 & 49 & 0.593 \\
\hline Body mass index (kg/m²) & $24.7 \pm 3.7$ & $24.4 \pm 3.0$ & $25.0 \pm 3.6$ & 0.400 \\
\hline Current smoking (\%) & 21 & 23 & 25 & 0.798 \\
\hline Habitual alcohol drinking (\%) & 26 & 22 & 28 & 0.611 \\
\hline Leisure-time physical activity (\%) & 67 & 52 & 49 & 0.023 \\
\hline Statin use (\%) & 14 & 32 & 42 & $<0.001$ \\
\hline NSAID use (\%) & 5 & 34 & 38 & $<0.001$ \\
\hline $\mathrm{SBP}^{\mathrm{a}}$ & $132 \pm 15$ & $134 \pm 18$ & $134 \pm 13$ & 0.398 \\
\hline $\mathrm{DBP}$ & $72 \pm 9$ & $75 \pm 11$ & $74 \pm 12$ & 0.068 \\
\hline $\mathrm{TG}(\mathrm{mmol} / \mathrm{L})^{\mathrm{a}}$ & $1.31(0.92,1.77)$ & $1.62(1.07,2.50)$ & $2.04(1.26,3.41)$ & $<0.001$ \\
\hline $\mathrm{TC}(\mathrm{mmol} / \mathrm{L})^{\mathrm{a}}$ & $4.83 \pm 0.90$ & $5.10 \pm 0.85$ & $4.94 \pm 1.09$ & 0.088 \\
\hline LDL-C (mmol/L) $)^{a}$ & $2.88 \pm 0.69$ & $3.08 \pm 1.06$ & $3.16 \pm 0.89$ & 0.082 \\
\hline $\mathrm{HDL}-\mathrm{C}(\mathrm{mmol} / \mathrm{L})^{\mathrm{a}}$ & $1.23 \pm 0.33$ & $1.16 \pm 0.41$ & $1.19 \pm 0.33$ & 0.306 \\
\hline Fasting plasma glucose (mmol/L) & $4.83 \pm 0.63$ & $8.62 \pm 1.70$ & $8.09 \pm 1.64$ & $<0.001$ \\
\hline Fasting insulin $(\mu \mathrm{U} / \mathrm{mL})$ & $6.32(5.17,8.77)$ & $8.43(7.09,10.88)$ & $7.83(5.99,11.06)$ & $<0.001$ \\
\hline HOMA-IR & $1.41(1.05,1.93)$ & $3.23(2.45,4.33)$ & $2.92(2.13,3.73)$ & $<0.001$ \\
\hline Duration of diabetes (years) & - & $6.7 \pm 3.1$ & $7.7 \pm 4.4$ & $<0.001$ \\
\hline $\mathrm{HbA1c}(\%)^{\mathrm{a}}$ & $5.1 \pm 0.4$ & $7.8 \pm 1.3$ & $7.7 \pm 1.0$ & $<0.001$ \\
\hline $\mathrm{HbA} 1 \mathrm{c}(\mathrm{mmol} / \mathrm{mol})^{\mathrm{a}}$ & $32.6 \pm 4.1$ & $62.0 \pm 14.1$ & $60.4 \pm 10.7$ & $<0.001$ \\
\hline $\mathrm{c}-\mathrm{IMT}(\mathrm{mm})^{\mathrm{a}}$ & $0.54 \pm 0.08$ & $0.74 \pm 0.09$ & $0.90 \pm 0.14$ & $<0.001$ \\
\hline ANGPTL $8^{\mathrm{a}}(\mathrm{pg} / \mathrm{mL})$ & $731 \pm 285$ & $1360 \pm 310$ & $1585 \pm 555$ & $<0.001$ \\
\hline
\end{tabular}

Data were expressed as mean \pm standard deviation, median (interquartile range), or percentage for continuous various and categorical variables, respectively. Cigarette smoking was defined as having smoked at least 100 cigarettes in one's lifetime. Regular leisure-time physical activity was defined as participation in $\geq 30$ min of moderate or vigorous activity per day at least 3 days per week

a Adjusted for age, gender, and BMI 
smoking, habitual alcohol drinking, SBP, DBP, TC, LDL-C and HDL-C among three groups.

\section{Correlation between ANGPTL8 and clinical parameters}

As shown by partial correlation analysis (Table 2 and Fig. 1), serum levels of ANGPTL8 were positively and significantly correlated with TG, duration of diabetes and c-IMT in type 2 diabetic patients with and without subclinical atherosclerosis groups (all $\mathrm{P}<0.05$ ) after adjustment for age, sex and BMI. No significant correlations were found between ANGPTL8 and SBP, DBP, TC, HDL-C, LDL-C, fasting plasma glucose, fasting insulin, HOMA-IR, HbA1c in all three groups. Multivariate regression analysis showed that TG, duration of diabetes and c-IMT were independently related factors influencing serum levels of ANGPTL8 in both type 2 diabetic patients with and without subclinical atherosclerosis groups (Table 3).

\section{Mediation analysis}

The mediation analysis revealed that TG partially mediated the positive relationship between ANGPTL8 and c-IMT in both type 2 diabetic patients with and without subclinical atherosclerosis groups (Fig. 2). When we tested the mediator role of TG in the relationship between ANGPTL8 and c-IMT in type 2 diabetic patients without subclinical atherosclerosis group (Fig. 2a), in the first linear regression equation, ANGPTL8 was positively associated with TG $(\mathrm{P}<0.001)$. In the second equation, ANGPTL8 was positively associated with c-IMT $(\mathrm{P}<0.001)$. In the third equation, when ANGPTL8 and TG were simultaneously included in the model, ANGPTL8 and TG were both positively associated with c-IMT (all $\mathrm{P}<0.05$ ). These data indicated that the effects of ANGPTL8 on c-IMT were partially mediated by TG. The Sobel Test for mediation estimated that the percentage of total effect (direct plus indirect) mediated by TG was $26.3 \%(\mathrm{z}=2.07 ; \mathrm{P}=0.037)$. The analysis of the mediator role of TG in the relationship between ANGPTL8 and c-IMT in type 2 diabetic patients with subclinical atherosclerosis group showed similar results (Fig. 2b), such that TG may be considered as partial mediator. The estimated percentage of total effect mediated by TG was $31.9 \%$ $(\mathrm{z}=2.29 ; \mathrm{P}=0.022)$. Because fasting plasma glucose, HbA1c and HOMA-IR were not related to ANGPTL8 significantly, the mediator roles of hyperglycemia and insulin resistance in the relationship between ANGPTL8 and c-IMT were not further evaluated.

\section{Associations between ANGPTL8 and subclinical atherosclerosis in type 2 diabetes}

Multivariate binary logistic regression revealed that serum ANGPTL8 was significantly related to subclinical atherosclerosis [odds ratio (OR) 3.05 (95\% CI 1.595.86), $\mathrm{P}=0.001$ ] without adjustment. Following multiple adjustment with age, sex, and body mass index (BMI), we observed that the OR for TG was significantly related to subclinical atherosclerosis [1.30 (95\% CI 1.04-1.63), $\mathrm{P}=0.023]$, while ANGPTL8 had the highest OR and was significantly related to subclinical atherosclerosis [OR 2.90 (95\% CI 1.48-5.70), $\mathrm{P}=0.002$ ] (Table 4).

Table 2 Correlations analysis of variables associated with circulating ANGPTL8 levels in control subjects with normal glucose tolerance (NGT), T2DM patients without atherosclerosis and T2DM patients with atherosclerosis

\begin{tabular}{|c|c|c|c|c|c|c|}
\hline & \multicolumn{2}{|c|}{ Control subjects with NGT } & \multicolumn{2}{|c|}{ T2DM without atherosclerosis } & \multicolumn{2}{|c|}{ T2DM with atherosclerosis } \\
\hline & $r$ & $\mathbf{P}$ & $\mathbf{r}$ & $\mathbf{P}$ & $r$ & $\mathbf{P}$ \\
\hline Age & 0.188 & 0.061 & 0.330 & 0.001 & 0.206 & 0.039 \\
\hline BMI & 0.000 & 0.997 & -0.029 & 0.778 & 0.235 & 0.018 \\
\hline SBPa & -0.031 & 0.763 & -0.042 & 0.686 & 0.106 & 0.301 \\
\hline DBPa & 0.126 & 0.218 & 0.033 & 0.746 & -0.037 & 0.723 \\
\hline $\mathrm{TG}^{\mathrm{a}}$ & 0.141 & 0.169 & 0.379 & $<0.001$ & 0.406 & $<0.001$ \\
\hline $\mathrm{TC}^{\mathrm{a}}$ & -0.055 & 0.596 & 0.020 & 0.848 & 0.083 & 0.419 \\
\hline LDL-C $C^{a}$ & -0.032 & 0.755 & 0.040 & 0.696 & -0.050 & 0.625 \\
\hline $\mathrm{HDL}-\mathrm{C}^{\mathrm{a}}$ & -0.144 & 0.158 & 0.088 & 0.392 & 0.075 & 0.466 \\
\hline Fasting plasma glucose ${ }^{a}$ & 0.127 & 0.216 & 0.087 & 0.395 & -0.009 & 0.932 \\
\hline Fasting insulin ${ }^{a}$ & -0.076 & 0.461 & 0.106 & 0.301 & 0.187 & 0.067 \\
\hline HOMA-IR & -0.028 & 0.789 & 0.114 & 0.265 & 0.160 & 0.117 \\
\hline $\mathrm{HbA} 1 \mathrm{c}^{\mathrm{a}}$ & 0.025 & 0.810 & 0.150 & 0.143 & -0.120 & 0.243 \\
\hline Duration of diabetes ${ }^{\mathrm{a}}$ & - & - & 0.250 & 0.013 & 0.306 & 0.002 \\
\hline $\mathrm{C}-\mathrm{IMT}(\mathrm{mm})^{\mathrm{a}}$ & 0.101 & 0.324 & 0.340 & 0.001 & 0.336 & 0.001 \\
\hline
\end{tabular}

a $\mathrm{P}$ value determined by partial correlation analysis (age, sex, and BMI adjusted) 

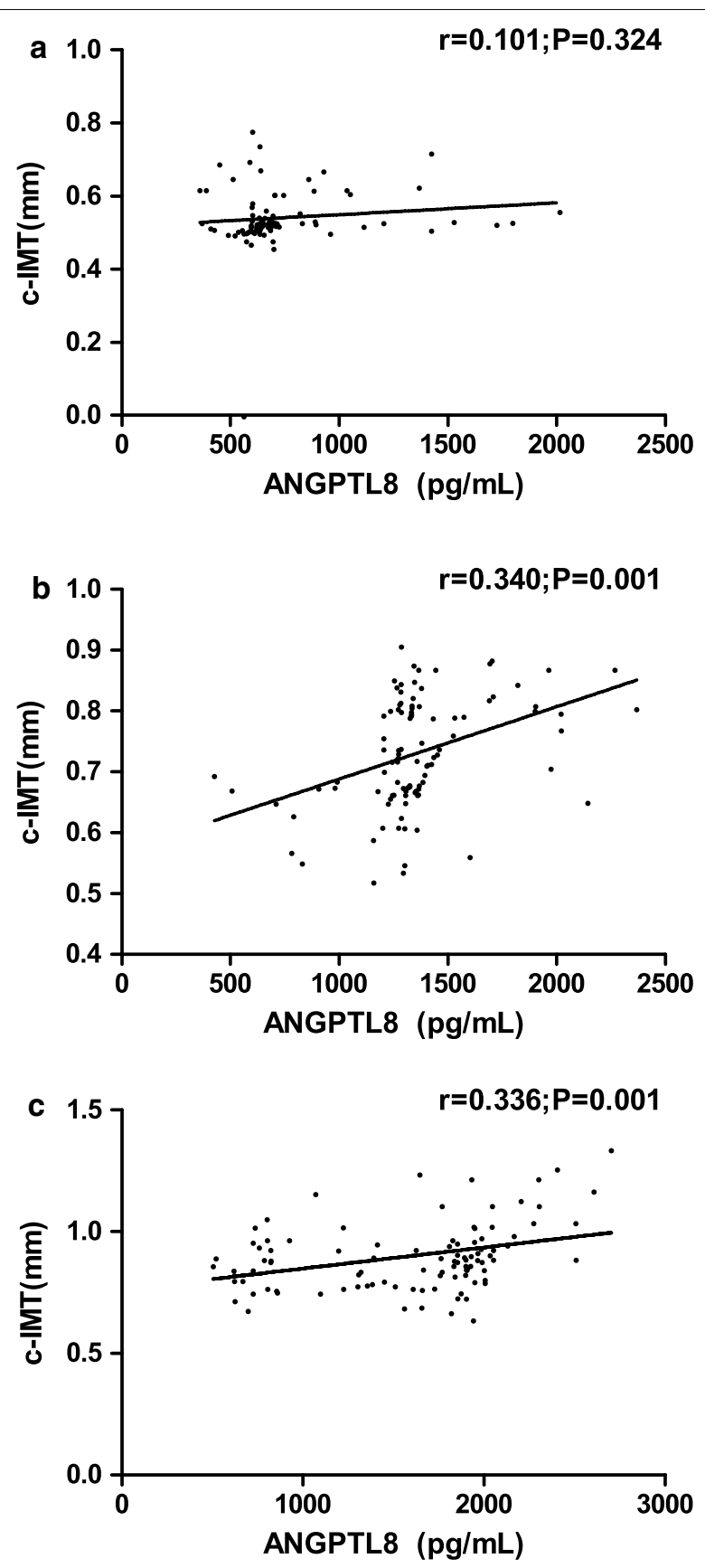

Fig. 1 a Correlations between ANGPTL8 and C-IMT in control subjects with normal glucose tolerance (NGT). b Correlations between ANGPTL8 and c-IMT in T2DM patients without atherosclerosis. c Correlations between ANGPTL8 and c-IMT in T2DM patients with atherosclerosis

\section{Discussion}

To our knowledge, the current study is the first to explore the potential mediating effect of hypertriglyceridemia, insulin resistance and hyperglycemia on the relationship between serum levels of ANGPTL8 and c-IMT and the possibility of identifying ANGPTL8 as a risk biomarker for subclinical atherosclerosis in type 2 diabetes through cross-sectional study in China. Four main results of the current study should be emphasized. First, serum levels of ANGPTL8 were increased in type 2 diabetic patients with subclinical atherosclerosis than in type 2 diabetic patients without subclinical atherosclerosis and control subjects. Second, increased serum levels of ANGPTL8 were positively associated with c-IMT in type 2 diabetes. Third, TG partially mediated this positive relationship between ANGPTL8 and c-IMT, whereas insulin resistance and hyperglycemia did not play a mediating role in this relationship. Fourth, ANGPTL8 might serve as a novel risk biomarker for subclinical atherosclerosis in type 2 diabetes.

There have been a number of studies showing increased circulating levels of ANGPTL8 in type 2 diabetic patients $[3,5,12,13]$ and also studies on unaltered or decreased circulating levels of ANGPTL8 [14, 15]. The reason for this discrepancy may be due to the difference in race, sample size, study design, medicine status, use of ELISA kits and handling of blood samples. In the current study, our data in agreement with most previous reports that serum levels of ANGPTL8 were increased in type 2 diabetic patients as compared with control subjects. In addition, we further found that serum levels of ANGPTL8 were significantly higher in type 2 diabetic patients with subclinical atherosclerosis group than that in type 2 diabetic patients without subclinical atherosclerosis group. Moreover, a positive association was found between ANGPTL8 and c-IMT in both type 2 diabetic patients with and without subclinical atherosclerosis groups. Although the causal relationship between ANGPTL8 and c-IMT can not be determined in this cross-sectional study, we still tried to investigate this relationship by asking what factors mediate this positive relationship between ANGPTL8 and c-IMT.

The relationship between ANGPTL8 and TG has well been established in animal models, mice lacking ANGPTL8 have significantly lower levels of TG than control mice [4], whereas overexpression of ANGPTL8 leads to increased levels of TG [16]. In agreement with these basic findings, our current data and other epidemiological evidence in humans indicated that serum levels of ANGPTL8 were positively and independently correlated with TG [17-19]. However, some other studies failed to find this significant correlation in type 2 diabetes $[12,20]$, the reason for this inconsistency might be partly attributed to the fact that many of the participants could have been treated with TG-lowering drugs which would affect TG levels, in our study, we excluded participants with any use of possible or known drugs affecting TG for more than 3 months or at any time within 12 months before the recruitment. In addition, mounting evidence 
Table 3 Multivariate regression analysis with ANGPTL8 as dependent variable

\begin{tabular}{|c|c|c|c|c|c|c|}
\hline & \multicolumn{2}{|c|}{ Control subjects with NGT } & \multicolumn{2}{|c|}{ T2DM without atherosclerosis ${ }^{a}$} & \multicolumn{2}{|c|}{$\begin{array}{l}\text { T2DM } \\
\text { with atherosclerosis }{ }^{a}\end{array}$} \\
\hline & $\beta$ & $\mathbf{P}$ & $\beta$ & $\mathbf{P}$ & $\beta$ & $\mathbf{P}$ \\
\hline SBPa & -0.032 & 0.763 & -0.040 & 0.686 & 0.103 & 0.301 \\
\hline $\mathrm{DBP}^{\mathrm{a}}$ & 0.132 & 0.218 & 0.033 & 0.746 & -0.035 & 0.723 \\
\hline $\mathrm{TG}^{\mathrm{a}}$ & 0.142 & 0.169 & 0.364 & $<0.001$ & 0.400 & $<0.001$ \\
\hline $\mathrm{TC}^{\mathrm{a}}$ & -0.055 & 0.596 & 0.019 & 0.848 & 0.080 & 0.419 \\
\hline LDL-C ${ }^{a}$ & -0.032 & 0.755 & 0.039 & 0.696 & -0.050 & 0.625 \\
\hline $\mathrm{HDL}-\mathrm{C}^{\mathrm{a}}$ & -0.142 & 0.158 & 0.083 & 0.392 & 0.072 & 0.466 \\
\hline Fasting plasma glucose $\mathrm{a}^{\mathrm{a}}$ & 0.125 & 0.216 & 0.083 & 0.395 & -0.008 & 0.932 \\
\hline Fasting insulin ${ }^{a}$ & -0.075 & 0.461 & 0.102 & 0.301 & 0.183 & 0.67 \\
\hline HOMA-IR & -0.027 & 0.789 & 0.109 & 0.265 & 0.158 & 0.117 \\
\hline $\mathrm{HbA} 1 \mathrm{c}^{\mathrm{a}}$ & 0.025 & 0.810 & 0.141 & 0.143 & -0.117 & 0.243 \\
\hline Duration of diabetes ${ }^{\mathrm{a}}$ & - & - & 0.242 & 0.013 & 0.294 & 0.002 \\
\hline $\mathrm{c}-\mathrm{IMT}(\mathrm{mm})^{\mathrm{a}}$ & 0.100 & 0.324 & 0.333 & 0.001 & 0.322 & 0.001 \\
\hline
\end{tabular}

${ }^{a}$ Age, sex, and BMl adjusted

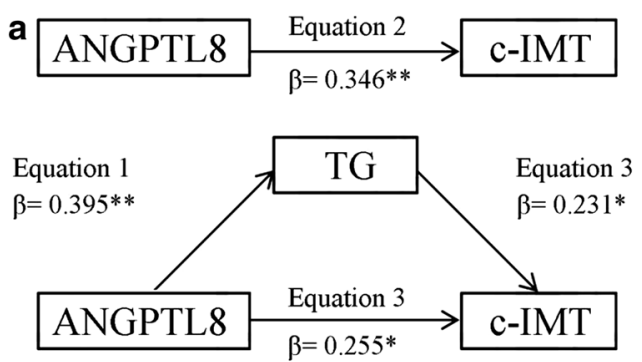

Sobel Test: $2.07 *(p=0.037)$
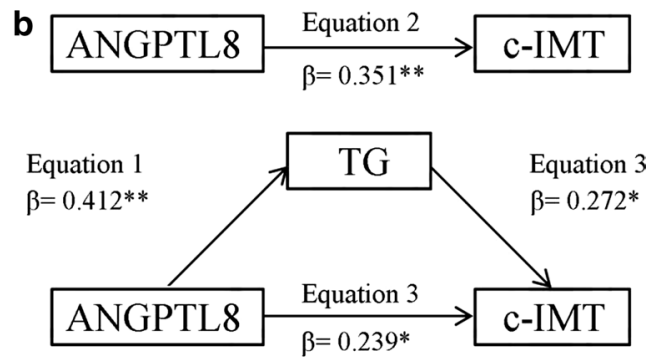

Sobel Test: $2.29 * \quad(\mathrm{p}=0.022)$

Fig. 2 a The mediator role of TG in the relationship between ANGPTL8 and c-IMT in type 2 diabetic patients without subclinical atherosclerosis group. b The mediator role of TG in the relationship between ANGPTL8 and c-IMT in type 2 diabetic patients with subclinical atherosclerosis group

suggested that TG may stimulate atherogenesis through production of proinflammatory cytokines, fibrinogen and coagulation factors and impairment of fibrinolysis $[21,22]$. Consistently, our data showed a positive correlation between TG and c-IMT. Our mediation analysis revealed that as ANGPTL8 increased, c-IMT increased, but when the influence of TG was controlled, the relationship between ANGPTL8 and c-IMT was mitigated, consequently, the influence of ANGPTL8 on c-IMT was partially mediated by TG. So one possible explanation for the relationship between ANGPTL8 and c-IMT is that ANGPTL8 is involved in the pathogenesis of atherosclerosis through regulating TG levels. Some evidence suggested that ANGPTL8 had also been linked to cholesterol metabolism [20], however, in this study, we did not find any significant association between ANGPTL8 and TC, HDL-C or LDL-C, this inconsistency might be partly attributed to the statin use in our study.

Aside from TG, ANGPTL8 had also been shown to be connected with blood glucose and insulin resistance $[5$,
12]. However, when we evaluated the possible relationship between ANGPTL8 and blood glucose or insulin resistance, no significant relationship was found between blood glucose, insulin resistance and ANGPTL8 in type 2 diabetes, this result corresponded with the observation by Fenzl et al. [20]. Notably, the significant relationship between blood glucose, insulin resistance and ANGPTL8 was mostly found in newly diagnosed type 2 diabetes [5, 12], whereas the nonsignificant relationship was mostly found in treated type 2 diabetes [20]. Consequently, it is speculated that antidiabetic treatments might, at least in part, lead to this obscure or nonsignificant relationship between blood glucose, insulin resistance and ANGPTL8 in type 2 diabetes. The mediator effects of fasting plasma glucose, HbA1c and HOMA-IR on the relationship between ANGPTL8 and c-IMT were not further evaluated because of this nonsignificant association.

Recent studies have confirmed a link between circulating levels of ANGPTL8 and cardiovascular disease related biomarkers such as metabolic syndrome and 
Table 4 OR $(95 \% \mathrm{Cl})$ by binary logistic regression models for atherosclerosis

\begin{tabular}{|c|c|c|c|c|c|}
\hline \multirow[t]{2}{*}{ Models } & \multirow[t]{2}{*}{ Covariates } & \multicolumn{2}{|c|}{$\begin{array}{l}\text { T2DM with- } \\
\text { out atheroscle- } \\
\text { rosis }\end{array}$} & \multicolumn{2}{|c|}{$\begin{array}{l}\text { T2DM with athero- } \\
\text { sclerosis }\end{array}$} \\
\hline & & OR $(95 \% \mathrm{Cl})$ & $P$ & OR $(95 \% \mathrm{Cl})$ & $\mathbf{P}$ \\
\hline \multirow[t]{4}{*}{ Model 1} & Age & 1.0 & - & $1.02(0.98,1.05)$ & 0.371 \\
\hline & $\mathrm{TG}$ & 1.0 & - & $1.34(1.08,1.67)$ & 0.009 \\
\hline & $\begin{array}{l}\text { Duration of dia- } \\
\text { betes }\end{array}$ & 1.0 & - & $1.07(0.99,1.15)$ & 0.082 \\
\hline & ANGPTL8 & 1.0 & - & $3.05(1.59,5.86)$ & 0.001 \\
\hline \multirow[t]{4}{*}{ Model 2} & Age & 1.0 & - & - & \\
\hline & $\mathrm{TG}$ & 1.0 & - & $1.33(1.06,1.67)$ & 0.013 \\
\hline & $\begin{array}{l}\text { Duration of dia- } \\
\text { betes }\end{array}$ & 1.0 & - & $1.07(0.99,1.15)$ & 0.102 \\
\hline & ANGPTL8 & 1.0 & - & $3.04(1.56,5.94)$ & 0.001 \\
\hline \multirow[t]{4}{*}{ Model 3} & Age & 1.0 & - & $1.02(0.98,1.05)$ & 0.398 \\
\hline & $\mathrm{TG}$ & 1.0 & - & $1.33(1.07,1.66)$ & 0.012 \\
\hline & $\begin{array}{l}\text { Duration of dia- } \\
\text { betes }\end{array}$ & 1.0 & - & $1.07(0.99,1.15)$ & 0.085 \\
\hline & ANGPTL8 & 1.0 & - & $2.99(1.56,5.75)$ & 0.001 \\
\hline \multirow[t]{4}{*}{ Model 4} & Age & 1.0 & - & $1.01(0.98,1.05)$ & 0.490 \\
\hline & $\mathrm{TG}$ & 1.0 & - & $1.32(1.05,1.65)$ & 0.016 \\
\hline & $\begin{array}{l}\text { Duration of dia- } \\
\text { betes }\end{array}$ & 1.0 & - & $1.07(0.99,1.16)$ & 0.071 \\
\hline & ANGPTL8 & 1.0 & - & $2.92(1.51,5.65)$ & 0.001 \\
\hline \multirow[t]{4}{*}{ Model 5} & Age & 1.0 & - & - & \\
\hline & $\mathrm{TG}$ & 1.0 & - & $1.30(1.04,1.63)$ & 0.023 \\
\hline & $\begin{array}{l}\text { Duration of dia- } \\
\text { betes }\end{array}$ & 1.0 & - & $1.07(0.99,1.15)$ & 0.087 \\
\hline & ANGPTL8 & 1.0 & - & $2.90(1.48,5.70)$ & 0.002 \\
\hline
\end{tabular}

Model 1 unadjusted, Model 2 age adjusted, Model 3 sex adjusted, Model 4 BMI adjusted, Model 5 age, sex, and BMI adjusted

inflammatory cytokines [23-26]. In three studies [23, 25, 26], for example, Abu-Farha et al., Crujeiras et al. and Liu et al. all found that circulating levels of ANGPTL8 were higher in participants with metabolic syndrome than those without, participants with higher ANGPTL8 levels experienced higher odds of having metabolic syndrome [23, 26]. With regard to the relationship between ANGPTL8 and inflammatory cytokines, Abu-Farha et al. also found a positive correlation between ANGPTL8 and HsCRP, suggesting that the inflammation might be connected to the increase in ANGPTL8 in humans that could result in aggravated cardiovascular disease. However, in the current study, the relationships between metabolic syndrome, inflammatory cytokines and ANGPTL8 were not assessed.

Binary logistic regression analysis was used to estimate the association of ANGPTL8 with the odds of subclinical atherosclerosis in type 2 diabetes, it revealed that elevated levels of ANGPTL8 were significantly associated with an increased risk of subclinical atherosclerosis in type 2 diabetes. Together our data may point towards a novel role for ANGPTL8 in the pathogenesis of atherosclerosis in type 2 diabetic patients, whereas in control subjects with normal glucose tolerance the potential deleterious effects of ANGPTL8 could still be compensated.

Despite the significant results of our current study, several limitations should be considered. First, this study is an epidemiological cross-sectional research and somehow fails to address the causal role of ANGPTL8 in the pathogenesis of subclinical atherosclerosis in type 2 diabetes, which is needed to be elucidated by further investigation. Second, the positive relationship between ANGPTL8 and c-IMT is probably related to more than a single mediator variable (TG), further research is still needed to clarify more potential mediator role of other unknown factors. Third, this study fails to investigate the relationships between ANGPTL8 and cardiovascular disease related biomarkers such as metabolic syndrome and inflammatory cytokines. Fourth, ANGPTL8 levels were not further quantified using different human ANGPTL8 kits to confirm the results of this study. Fifth, generalizations based on our results could be limited because of unmeasured confounders.

\section{Conclusions}

In summary, results of the current study revealed that serum levels of ANGPTL8 were significantly increased in type 2 diabetic patients with subclinical atherosclerosis, moreover, our data provided the first evidence that ANGPTL8 was positively correlated with c-IMT in this population and TG partially mediated this positive correlation, suggesting ANGPTL8 to be a new biomarker for subclinical atherosclerosis in type 2 diabetes. Further studies are needed to validate the causative relationship between ANGPTL8 and subclinical atherosclerosis in type 2 diabetes.

\section{Abbreviations}

ANGPTL8: angiopoietin-like protein 8; BMI: body mass index; c-IMT: common carotid artery Intima-Media Thickness; FPG: fasting plasma glucose; HOMA-IR: homeostasis model assessment of insulin resistance; HDL-C: high-density lipoprotein-cholesterol; LDL-C: low-density lipoprotein-cholesterol; NGT: normal glucose tolerance; OR: odds ratio; RIFL: refeeding-induced fat and liver; TG: triglyceride; TC: total cholesterol.

\section{Authors' contributions}

$\mathrm{ZTP}, \mathrm{GB}, \mathrm{LHB}, \mathrm{CB}, \mathrm{XLP}$ and SJF contributed to the study concept and design. GB, SJF, LHB and XLP contributed the data. ZTP and QLY planned the statistical analysis. QLY conducted the statistical analysis. ZTP, GB and LHB drafted the paper. All authors contributed to, critically revised. All authors read and approved the final manuscript.

\section{Author details}

${ }^{1}$ Department of Endocrinology and Metabolism, The Second Affiliated Hospital of Guilin Medical University, 212 Renmin Road, Guilin 541199, Guangxi, People's Republic of China. ${ }^{2}$ Department of Urology, The Second 
Affiliated Hospital of Guilin Medical University, Guilin, Guangxi, People's Republic of China. ${ }^{3}$ Department of Laboratory Medicine, The Second Affiliated Hospital of Guilin Medical University, Guilin, Guangxi, People's Republic of China. ${ }^{4}$ Department of Human Anatomy, Southwest Medical University, Luzhou, Sichuan, People's Republic of China. ${ }^{5}$ Department of Epidemiology and Health Statistics, Guilin Medical University, Guilin, Guangxi, People's Republic of China. ${ }^{6}$ Department of Thoracic and Cardiovascular Surgery, The Second Affiliated Hospital of Guilin Medical University, Guilin, Guangxi, People's Republic of China.

\section{Acknowledgements}

The authors wish to thank each of the participant for the data provided.

\section{Competing interests}

The authors declare that they have no competing interests.

\section{Availability of data and materials}

The datasets during and/or analyzed during the current study are available from the corresponding author with reasonable request.

\section{Consent for publication}

Participants were informed of data sharing with their name and identity hidden per consent.

\section{Ethics approval and consent to participate}

This study was approved by the Ethics Committee of Affiliated Hospital of Guilin Medical University. All participants provided written informed consent.

\section{Funding}

This study was supported by grants from National Natural Science Foundation of China (Grant Nos. 81770829 and 81560144), Scientific Research and Technology Development Projects of Science and Technology Department of Guangxi province (Grant No. 1598012-13) and Natural Science Foundation of Guangxi province (Grant No. 2015GXNSFBA139119).

\section{Publisher's Note}

Springer Nature remains neutral with regard to jurisdictional claims in published maps and institutional affiliations.

Received: 7 December 2017 Accepted: 17 March 2018 Published online: 15 July 2018

\section{References}

1. Zheng TP, Liu YH, Yang LX, Qin SH, Liu HB. Increased plasma dipeptidyl peptidase- 4 activities are associated with high prevalence of subclinical atherosclerosis in Chinese patients with newly diagnosed type 2 diabetes: a cross-sectional study. Atherosclerosis. 2015;242(2):580-8.

2. Beckman JA, Creager MA, Libby P. Diabetes and atherosclerosis: epidemiology, pathophysiology, and management. JAMA. 2002;287(19):2570-81.

3. Yue S, Wu J, Zhang J, Liu L, Chen L. The relationship between betatrophin levels in blood and T2DM: a systematic review and meta-analysis. Dis Markers. 2016;2016:9391837.

4. Wang Y, Quagliarini F, Gusarova V, Gromada J, Valenzuela DM, Cohen JC, Hobbs HH. Mice lacking ANGPTL8 (Betatrophin) manifest disrupted triglyceride metabolism without impaired glucose homeostasis. Proc Natl Acad Sci USA. 2013;110(40):16109-14.

5. Chen X, Lu P, He W, Zhang J, Liu L, Yang Y, Liu Z, Xie J, Shao S, Du T, et al. Circulating betatrophin levels are increased in patients with type 2 diabetes and associated with insulin resistance. J Clin Endocrinol Metab. 2015;100(1):E96-100.

6. Gomez-Ambrosi J, Pascual-Corrales E, Catalan V, Rodriguez A, Ramirez B, Romero S, Vila N, Ibanez P, Margall MA, Silva C, et al. Altered concentrations in dyslipidemia evidence a role for ANGPTL8/betatrophin in lipid metabolism in humans. J Clin Endocrinol Metab. 2016;101(10):3803-11.

7. Zheng T, Qin L, Chen B, Hu X, Zhang X, Liu Y, Liu H, Qin S, Li G, Li Q. Association of plasma DPP4 activity with mild cognitive impairment in elderly patients with type 2 diabetes: results from the GDMD study in China. Diabetes Care. 2016;39(9):1594-601.

8. Yang W, Lu J, Weng J, Jia W, Ji L, Xiao J, Shan Z, Liu J, Tian H, Ji Q, et al. Prevalence of diabetes among men and women in China. N Engl J Med. 2010;362(12):1090-101.

9. Zheng TP, Yang F, Gao Y, Baskota A, Chen T, Tian HM, Ran XW. Increased plasma DPP4 activities predict new-onset atherosclerosis in association with its proinflammatory effects in Chinese over a 4 year period: a prospective study. Atherosclerosis. 2014;235(2):619-24.

10. Baron RM, Kenny DA. The moderator-mediator variable distinction in social psychological research: conceptual, strategic, and statistical considerations. J Pers Soc Psychol. 1986;51(6):1173-82.

11. Sobel M. Asymptotic confidence interval for indirect effects in structural equation models. In: Leinhardt S, editor. Sociological Methodology. Washington, DC: American Sociological Association; 1982. p. 290-312.

12. Hu H, Sun W, Yu S, Hong X, Qian W, Tang B, Wang D, Yang L, Wang J, Mao $C$, et al. Increased circulating levels of betatrophin in newly diagnosed type 2 diabetic patients. Diabetes Care. 2014;37(10):2718-22.

13. Espes D, Martinell M, Liljeback H, Carlsson PO. Betatrophin in diabetes mellitus: the epidemiological evidence in humans. Curr DiabRep. 2015;15(12):104.

14. Gomez-Ambrosi J, Pascual E, Catalan V, Rodriguez A, Ramirez B, Silva C, Gil MJ, Salvador J, Fruhbeck G. Circulating betatrophin concentrations are decreased in human obesity and type 2 diabetes. J Clin Endocrinol Metab. 2014;99(10):E2004-9.

15. Guo K, Lu J, Yu H, Zhao F, Pan P, Zhang L, Chen H, Bao Y, Jia W. Serum betatrophin concentrations are significantly increased in overweight but not in obese or type 2 diabetic individuals. Obesity. 2015;23(4):793-7.

16. Zhang R. Lipasin, a novel nutritionally-regulated liver-enriched factor that regulates serum triglyceride levels. Biochem Biophys Res Commun. 2012;424(4):786-92.

17. Chen CC, Susanto H, Chuang WH, Liu TY, Wang CH. Higher serum betatrophin level in type 2 diabetes subjects is associated with urinary albumin excretion and renal function. Cardiovasc Diabetol. 2016;15:3.

18. Wang $Y Y$, Zhang D, Jiang $Z Y$, Lu XQ, Zheng $X, Y u Y$ J, Wang YG, Dong J, Positive association between betatrophin and diabetic retinopathy risk in type 2 diabetes patients. Horm Metab Res $=$ Hormon- und Stoffwechselforschung $=$ Hormones et metabolisme. 2016;48(3):169-73.

19. Gao T, Jin K, Chen P, Jin H, Yang L, Xie X, Yang M, Hu C, Yu X. Circulating betatrophin correlates with triglycerides and postprandial glucose among different glucose tolerance statuses-A case-control study. PLoS ONE. 2015;10(8):e0133640.

20. Fenzl A, Itariu BK, Kosi L, Fritzer-Szekeres M, Kautzky-Willer A, Stulnig TM, Kiefer FW. Circulating betatrophin correlates with atherogenic lipid profiles but not with glucose and insulin levels in insulin-resistant individuals. Diabetologia. 2014;57(6):1204-8.

21. Tenenbaum A, Klempfner R, Fisman EZ. Hypertriglyceridemia: a too long unfairly neglected major cardiovascular risk factor. Cardiovasc Diabetol. 2014; 13:159.

22. Kim MK, Ahn CW, Kang S, Nam JS, Kim KR, Park JS. Relationship between the triglyceride glucose index and coronary artery calcification in Korean adults. Cardiovasc Diabetol. 2017;16(1):108.

23. Abu-Farha M, Abubaker J, Al-Khairi I, Cherian P, Noronha F, Kavalakatt S, Khadir A, Behbehani K, Alarouj M, Bennakhi A, et al. Circulating angiopoietin-like protein 8 (betatrophin) association with HsCRP and metabolic syndrome. Cardiovasc Diabetol. 2016;15:25.

24. Abu-Farha M, Abubaker J, Tuomilehto J. ANGPTL8 (betatrophin) role in diabetes and metabolic diseases. Diabetes metab Res Rev. 2017;33(8):e2919-27.

25. Crujeiras AB, Zulet MA, Abete I, Amil M, Carreira MC, Martinez JA, Casanueva FF. Interplay of atherogenic factors, protein intake and betatrophin levels in obese-metabolic syndrome patients treated with hypocaloric diets. Int J Obes. 2016;40(3):403-10.

26. Liu D, Li S, He H, Yu C, Li X, Liang L, Chen Y, Li J, Li J, Sun X, et al. Increased circulating full-length betatrophin levels in drug-naive metabolic syndrome. Oncotarget. 2017;8(11):17510-7. 